\title{
Tatbild und Tatanalyse
}

\author{
Hans-Ludwig Kröber
}

Online publiziert: 26. März 2010

(C) Springer-Verlag 2010

Tatbild und Tatanalyse sind das Schwerpunktthema dieses Heftes. Dabei sind drei Beiträge der operativen Fallanalyse (OFA) gewidmet: Ihre grundsätzliche Methodik wird von Dern dargestellt, Erpenbach verdeutlicht die Arbeitsweise an einem Fallbeispiel, und Boetticher veranschaulicht aus Sicht des Bundesrichters, welche Bedeutung die OFA im Gerichtssaal gewinnen kann - und welche nicht. Tatsächlich wird hier, am Ende des kriminalistischen Arbeitsprozesses, sichtbar, dass es interessante Parallelen zwischen den Beurteilungsprozessen der Kriminalbeamten und der Richter gibt. Sobald aber die Hauptverhandlung begonnen hat, geht es um Zuarbeit, um Materiallieferung für den gerichtlichen Bewertungs- und Entscheidungsprozess. Zuvor jedoch ist OFA teamförmige, systematische Ermittlungsarbeit bei der Suche nach dem Täter, in die bisweilen nicht nur Rechtsmediziner, sondern auch forensische Psychiater und/ oder Rechtspsychologen involviert werden, um anhand des Spurenbilds Hypothesen zu prüfen, wie jene, ob der Täter psychisch gestört war, alt oder jung, Mann oder Frau, ob sich eine bestimmte Motivation oder auch Obsession aufdrängt. Die Hypothesenprüfung allein anhand einer Tatortanalyse, ohne einen Tatverdächtigen, verdeutlicht gerade dem Psychiater, wie weit bisweilen die Abstände zwischen einem Persönlichkeitsprofil und einem Tatbild sein mögen. Eine stimmige Zuordnung zwischen beiden - die Tat passt zum Täter - vermag man oft erst im Nachhinein zu sehen, während man zuvor noch ganz andere Tätertypen nicht ausschließen mochte. Vermeintliche Zwangsläufigkeiten - so

Prof. Dr. med. H.-L. Kröber $(\bowtie)$

Institut für Forensische Psychiatrie der Charité -

Universitätsmedizin Berlin, Limonenstr. 27,

12203 Berlin, Deutschland

E-Mail: Hans-Ludwig.Kroeber@charite.de ein Täter musste ja so eine Tat begehen - erweisen sich dann als ,,bias“, als Effekt der nachträglichen Verknüpfung; prospektiv hätte man nicht zu sagen gewagt, dass dieser Täter diese Tat begehen muss und kein anderer Typ sie begehen könnte. Insofern ist OFA eine Form der Eingrenzung und Optimierung der Suche, während die Überführung des Täters im Einzelfall dann schließlich - nach dieser Vorarbeit - mit klassischen kriminalistischen Mitteln erfolgt, am liebsten durch ein Geständnis wie im Leipziger Fall Michelle; hier lag die OFA völlig richtig, hätte aber fast keine Beweismittel gegen den Täter gehabt.

Besondere Bedeutung hat die genaue Tatanalyse bei Gewalt- und Sexualstraftätern, und gerade die Annahme einer sadistischen Motivation hat oft weitreichende Folgen für die Gefährlichkeitseinschätzung und für die Dauer von Freiheitsentziehung. Berner u. Briken beschreiben Ansätze, um hier zu einer sicheren Diagnostik anhand der wahrnehmbaren Fakten zu kommen. Dahle et al. verdeutlichen in ihrem Beitrag, dass tatsächlich die Erfassung allein von Geschehensabläufen sexueller Straftaten eine relevante kriminalprognostische Aussage ermöglicht; zugleich ist dies eine Evaluation von Tatmerkmalen, die im Einzelfall für bedeutsam gehalten werden. Die Tatbildanalysen werden schließlich durch den Beitrag von Prüter abgerundet, der verdeutlicht, dass bei gleichem Krankheitsbild einer Schizophrenie eine Reihe von ganz unterschiedlichen Motivationen und Tatkonstellationen auftreten kann, die wiederum Konsequenzen für die Gefährlichkeitseinschätzung und die Therapie haben. Moderne forensische Psychiatrie zeichnet sich dadurch aus, dass sie auch von kriminologischem Wissen getränkt ist und Tatbilder dadurch nicht nur fantasiereich psychodynamisch deuten, sondern im kriminellen Spektrum und im Hinblick auf die Prognose einordnen kann. 